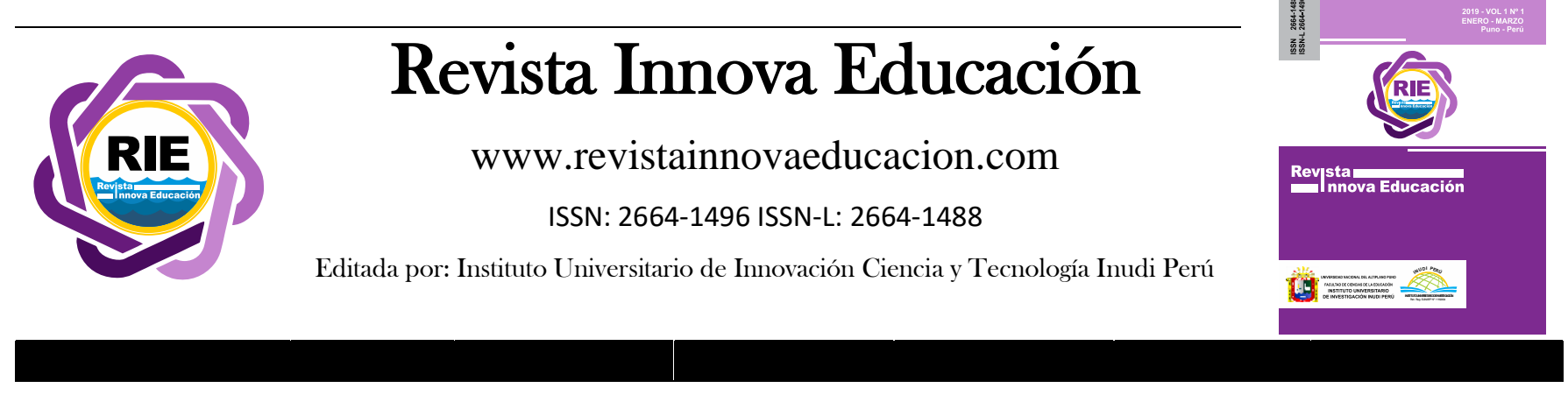

\title{
Los inicios del conocimiento didáctico tecnológico del contenido en las adscripciones universitarias. Estudio de casos
}

\author{
The beginning of the Technological Pedagogical Content Knowledge in assistant student to the \\ university. Study of cases
}

Mara Moreyra (D); Patricia Demuth $(1 D$

DOI: https://doi.org/10.35622/j.rie.2019.04.006

${ }^{1}$ Universidad Nacional del Nordeste, Argentina.

Recibido el 24/09/2019/ Aceptado el 19/10/2019/

\begin{abstract}
ARTÍCULO ORIGINAL
Estudio de casos.

PALABRAS CLAVE

Adscripción, competencias, principiantes, tecnología educativa, universidad.

Este trabajo tiene por objetivo comprender los procesos de Construcción del Conocimiento Didáctico Tecnológico del Contenido en principiantes adscriptos universitarios, considerándolos docentes en formación, en el contexto de la Universidad Nacional del Nordeste, Argentina. Teniendo en cuenta el contexto universitario, buscamos profundizar y ampliar el análisis de tres casos provenientes de distintas carreras de las facultades de dicha universidad: Facultad de Medicina, Ciencias Exactas y Naturales y Agrimensura y Humanidades; cuyas carreras y asignaturas son: Medicina (Bioquímica), Profesorado de Biología (Biología de los Cordados) y Licenciatura en Ciencias de la Educación (Elaboración y Evaluación de Proyectos).Dado el tipo de análisis que procuramos, enmarcamos dicha investigación dentro del paradigma interpretativo y enfoque cualitativo. Asimismo, la metodología adoptada fue el estudio de casos, cuyos instrumentos de recogida de información fueron sucesivas entrevistas en profundidad, semi-estructuradas individuales; observaciones de sus prácticas de adscripción, materiales curriculares y proyectos de adscripción de los sujetos. En cuanto a las conclusiones señalamos a la adscripción universitaria como un campo de formación profesional complejo, enriquecido de conocimientos y experiencias que permiten desplegar una serie de competencias iniciales en los principiantes, las cuales se agrupan en competencias convencionales y competencias emergentes.
\end{abstract}

\section{KEYWORDS}

Ascription, competitions, beginners, educational technology, college.
This work aims to understand the processes of Construction of the Technological Didactic Knowledge of the Content in beginner university affiliates, considering them as teachers in training, in the context of the National University of the Northeast, Argentina. Taking into account the university context, we seek to deepen and broaden the analysis of three cases from different careers of the faculties of said university: Faculty of Medicine, Exact and Natural Sciences and Surveying and Humanities; whose careers and subjects are: Medicine (Biochemistry), Faculty of Biology (Biology of the Ropes) and Bachelor of Science in Education (Project Development and Evaluation). Given the type of analysis we seek, we frame this research within the interpretive paradigm and qualitative approach. Likewise, the methodology adopted was the case study, whose information collection instruments were successive in-depth interviews, individual semi-structured; Observations of their ascription practices, curricular materials and projects of ascription of the subjects. Regarding the conclusions, we point to university ascription as a complex vocational training field, 
enriched with knowledge and experiences that allow the deployment of a series of initial skills in beginners, which are grouped into conventional and emerging skills.

\section{INTRODUCCIÓN}

Este proyecto de investigación tuvo sus inicios en el proyecto de investigación "La Construcción del Conocimiento Didáctico del Contenido (CDC) en profesores experimentados y principiantes de la Universidad Nacional del Nordeste. Estudio de casos múltiples", (PI H010- 2014. Resol.: 984/14). Asimismo, se trabajó con antecedentes bibliográficos que sirvieron como base y fueron seleccionados por el equipo de investigación mayor como aquellos utilizados y propios del Grupo de investigación CyFOD- Conocimiento y Formación Docente- integrado por docentes de diversas cátedras de la Facultad de Humanidades y de la Facultad Ciencias Exactas, Naturales y Agrimensura; la especificidad del objeto de estudio, orientó la búsqueda de material relativo a los sujetos de investigación, la figura del estudiante/principiante adscripto universitario, que adquiere distintas connotaciones, tanto en las facultades seleccionadas para su análisis, como en otras universidades del país; se menciona a la Universidad de Buenos Aires y Universidad Nacional de Mar del Plata; (Solberg, Droblas, Rodríguez, Ulloa y Viñas, 2007; Ferreiro y Linch, 2015), quienes interpretan este trayecto o instancia como momentos y espacios de formación para el futuro docente novel.

Trabajar en el análisis de la formación docente focalizando en el conocimiento docente permite ingresar y tomar contacto con la complejidad de este, su particular conformación y la articulación efectiva de retroalimentación que tiene con la práctica de enseñanza. Ésta se encuentra orientada por las creencias y conocimientos que poseen los profesores sobre la enseñanza, los estudiantes y docentes, los contenidos a enseñar, como las propias experiencias de formación que generan diversos aprendizajes.

Es aquí que se identifica y recupera el periodo formativo del futuro profesor que se produce entre su formación inicial y su futuro desarrollo profesional. El llamado "periodo de inserción", que actúa como un eslabón y nexo entre ambos. El mismo hace referencia por ejemplo a cursos, capacitaciones, pasantías, adscripciones en asignaturas, experiencias vinculadas a la realidad educativa que tienen los futuros profesores. Estas últimas espacios únicos y trascendentales para la formación en la cual el futuro docente va construyendo su conocimiento profesional, presentan particularidades distintivas, dependiendo de su contexto.

Se destaca el estudio realizado por Torres y Arras (2011), quienes analizan el conocimiento didáctico tecnológico del contenido y las competencias a desarrollar en la formación de pregado. Si bien, es un trabajo de tipo cuantitativo y de comparación, es posible recuperar ciertas nociones que brindaron insumos en el diseño de los instrumentos de recolección de datos y el análisis posterior de los mismos. Tales como, las diversas categorizaciones del concepto de "competencias" en el uso productivo de las Nuevas Tecnologías de la Información y la Comunicación (TIC), como "las competencias básicas", "de aplicación y profundización", "éticas" y las tecnologías más utilizadas en el nivel superior universitario. Permitieron pulir los 
interrogantes en torno a el Conocimiento Didáctico Tecnológico del Contenido (CDTC) y la nueva variable con la que se trabajó, las competencias en las instancias de formación de pregrado y grado.

Los estudios que refieren a esta línea de investigación perteneciente al campo de la didáctica de nivel superior con relación al conocimiento profesional docente se diferencian teniendo en cuenta los antecedentes del proyecto en el que se inscribe (principalmente investigaciones realizadas por miembros del equipo) y los autores más destacados. Estos han realizado aportes fundamentales para el análisis y comprensión del conocimiento de profesores, que según Briones (2001) incluye las ideas para desarrollar la clase, convicciones, creencias sobre el comportamiento de sus estudiantes; la búsqueda del conocimiento experto (sus características, las características del profesor principiante); el conocimiento reflexivo (la investigación acción en el aula y la escuela); conocimiento didáctico del contenido (transformación del contenido didáctico de la disciplina en contenidos enseñables, adecuación metodológica del contenido).

Asimismo, Montero (2001) señala que la importancia de profundizar en el estudio del proceso de construcción del conocimiento profesional docente recae en la posibilidad para repensar la función docente y la formación del profesorado, reconociendo que este interés se halla motivado por factores prácticos, políticos y académicos. Otra de las categorías fundamentales de análisis, es "la construcción de un conocimiento base para la enseñanza", analizada desde los aportes de Shulman (1987), quien plantea los conocimientos necesarios para la enseñanza, como el conocimiento del contenido, el didáctico general, del currículo, didáctico del contenido, el de los estudiantes y contextos educativos, entre otros.

Si bien los inicios de los estudios sobre el constructo CDC se atribuyen al autor mencionado, Shulman (1986-1987) fue a partir de los aportes de Koehler y Mishra (a partir del año 2005), quienes introdujeron el TPACK (Technological Pedagogical Content Knowledge), o CDTC), al entender que los docentes van transformando continuamente los conocimientos para convertirlos en didácticamente enseñables, competencia dominada por docentes expertos y sobre todo por aquellos que utilizan las tecnologías como parte del proceso de enseñanza y aprendizaje, en que la dimensión tecnológica adquiere relevancia.

Los autores que impulsaron la génesis y desarrollo del TPACK fueron Koehler y Mishra (2007) y Schmidt, Sahin, Thompson y Seymour (2008). Èste es un modelo de formación que pretende comprender la integración de distintos tipos de conocimientos, vinculados a los contenidos disciplinares, a la pedagogía y la tecnología, considerados necesarios para la incorporación eficaz de las TIC en las prácticas de enseñanza. Una interpretación reconocida sobre el mismo, es la siguiente:

El TPACK sugiere que los profesores han de tener un conocimiento tecnológico sobre cómo funcionan (...) las TIC tanto de forma general como de manera específica, además de saber la manera de cómo y en qué emplearlas; también deben poseer un conocimiento pedagógico, respecto a cómo enseñar eficazmente y, por último, un conocimiento sobre el contenido o disciplina respecto a la materia que deben enseñar. (Cabero y Garrido, 2015:14). 
Además, analizar el periodo formativo del profesor principiante que se produce entre su formación inicial y su futuro desarrollo profesional (periodo de inserción), es sumamente relevante para comprender la construcción de sus conocimientos. El mismo hace referencia por ejemplo a cursos, capacitaciones, experiencias con la realidad educativa que tienen los futuros profesores y es aquí donde se ubica la adscripción, como un espacio de formación donde el profesor en formación va construyendo ese conocimiento profesional docente. Las adscripciones permiten sumergir al futuro profesor a la realidad, organización, gestión de la práctica docente, ubicándose en la tríada didáctica en un espacio que actúa como nexo entre los estudiantes, contenidos y el profesor; posibilitándole éste el despliegue de diversas competencias, habilidades, conocimientos adquiridos durante su trayecto de formación y la construcción de nuevos saberes o conocimientos inherentes al contenido disciplinar y didáctico de la propia asignatura donde se encuentran insertos, conduciéndonos al CDC.

Al mismo tiempo, en líneas generales, Cukierman y Virgili (2013) realizan una descripción del contexto actual con respecto a las TIC y la educación en el nivel superior circunscribiéndose en Argentina, en la universidad Tecnológica Nacional denotando características particulares contextuales y rasgos de la formación específica en el uso de recursos tecnológicos. Sintetizan la situación presente del sistema educativo y las nuevas tecnologías en general; explican que la forma de enseñar actual no muestra una diferencia con las prácticas docentes del pasado y cómo esto colisiona con las características particulares de los estudiantes en la actualidad. Una cuestión que emerge a partir de esta reflexión, es si la formación en TIC de los estudiantes se centra en competencias, habilidades, destrezas que promueven un uso educativo de las mismas y bien solo se reduce al uso de la tecnología por la tecnología (Cabero Almenara y Marín Diaz 2017).

Asimismo, en la relación entre ciencia, tecnología y sociedad, predomina en el imaginario colectivo la perspectiva determinista tecnológica señalada por Ronderos y Valderrama (2003), quienes sostienen que se encuentra implícita en las formas de pensar, argumentando que los avances tecnológicos son los protagonistas y causantes de los cambios socioculturales. A su vez, se encuentra tan arraigado en el discurso social y fue inserto intencionalmente por sujetos que detentan el poder, quienes sostienen intereses particulares para reproducir este discurso.

Es fundamental reconocer entonces, un tejido con hilos imbricados entre la sociedad, la ciencia y la tecnología. Éstas integran una compleja red de interrelaciones entre sí y no sería justo ponderar el impacto solamente de las tecnologías sobre la esfera social y en consecuencia la trasformación radical del campo educativo ya que, es palpable en la realidad que esto no sucede de manera lineal. Aunque se reconoce que es necesario la sensibilización y concientización de los docentes con respecto a la integración de éstas en el diseño curricular, también se señala que la innovación en las aulas no se vincula con el uso del último recurso tecnológico digital de moda, sino con la aplicación de criterios para conseguir nuevos escenarios formativos y comunicativos, es decir en diseñar modelos de enseñanza para extraer de los recursos que utilizamos, el máximo provecho (Cabero y Diaz, 2017).

Por ello se incursiona en esta línea del TPACK (Technological Pedagogical Content Knowledge), que comienza a gestarse en los Estados Unidos, a partir de los estudios de Shulman, 
elaborándose nuevas construcciones teóricas sobre el conocimiento profesional docente, una de ellas, encabezada por Harris, Koehler y Mishra (2005, 2006, 2007, 2008), quienes retoman los aportes del primero pero avanzan en su actualización, teniendo en cuenta la transformación que sufre el campo educativo, particularmente a partir del año 2000 con la influencia de la TIC. Se focalizan en el estudio de la integración de las TIC en la enseñanza y proponen integrar al conocimiento sobre la tecnología, como una dimensión de conocimiento que se interrelaciona con el conocimiento pedagógico y el conocimiento disciplinar, dando lugar al surgimiento de otros nuevos. Entre ellos el TPACK, traducido al castellano como "conocimiento didáctico tecnológico del contenido" (CDTC), que se localiza al interior del constructo del CDC y que influencia en las prácticas docentes. Éste emerge a partir de la combinación de tres tipos de conocimientos que lo integran: Conocimiento Disciplinar (CK, Content Knowledge), y el Conocimiento Tecnológico (TK, Technological Knowledge) y Conocimiento Pedagógico (PK, Pedagogical) y que, a su vez integrados entre sí, dan lugar al Conocimiento Tecnológico Pedagógico (TPK), Conocimiento Tecnológico Disciplinar (TCK), Conocimiento Pedagógico disciplinar (PCK). Dicho grado de combinación entre éstos, se traducirá en mayor o menor nivel de integración de tecnologías en la enseñanza, por parte del docente, en un contexto determinado.

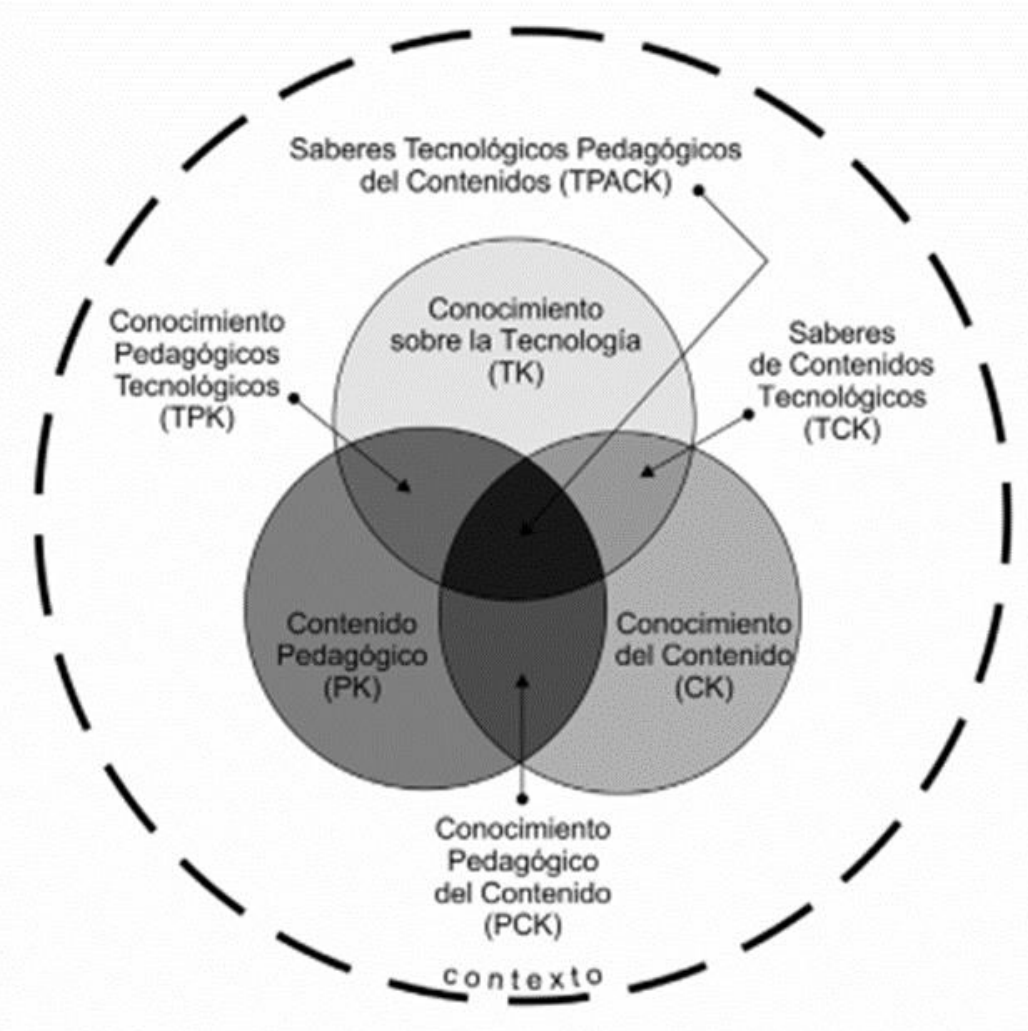

Figura 1.1. Esquema del modelo TPACK: disposición de los tres tipos de conocimientos básicos (Conocimiento Tecnológico, Pedagógico y Disciplinar) y las intersecciones correspondientes, generando, así, siete tipos de conocimientos. Reproducido de "Technological Pedagogical Content Knowledge: A new framework for teacher knowledge” p.1025, por Mishra, P., y Koheler, M. J. 2006, Teachers: College Record, 108(6), 1017-1054. 
De manera sintética y a fin de clarificar en qué consiste cada uno se describe a continuación, teniendo en cuenta la clasificación realizada por Mishra y Koheler (2006):

CK (Conocimiento del Contenido): se refiere a aquellos saberes que posee y elabora el docente sobre el conocimiento disciplinar que enseña, como conceptos, paradigmas, teorías, cuerpos teóricos, prácticas y evidencias.

TK (Conocimiento sobre la Tecnología): apunta al conocimiento sobre el manejo y uso de las TIC, como herramientas digitales o recursos tecnológicos disponibles, que tienen los docentes.

PK (Conocimiento Pedagógico): alude al conocimiento general sobre las características propias de los procesos de enseñanza y aprendizaje. Incluye a los objetivos, a las estrategias de enseñanza, técnicas, criterios e instrumentos de evaluación, entre otros.

PCK (Conocimiento pedagógico del Contenido): se traduce como la particular reinterpretación, construcción y deconstrucción que realiza el docente sobre los contenidos de su disciplina, logrando explicarlos, de forma más comprensible y entendible para sus estudiantes. A partir de las interpretaciones y representaciones que el docente ejerce sobre el contenido disciplinar, logra transformarlo, encontrar diversas maneras de presentarlo, explicarlo, vincularlo con otros conocimientos y los previos del estudiantado, seleccionar diversas estrategias de enseñanza para exponerlos, diseñar evaluaciones adecuadas, dando lugar a una flexibilidad didáctica.

TCK (Saberes de Contenidos Tecnológicos): es el conocimiento que tiene el docente sobre qué tipo de recursos tecnológicos guardan mayor utilidad para representar el tipo de contenido disciplinar que debe enseñar.

TPK (Conocimiento Tecnológico Pedagógico): se refiere al conocimiento no solo sobre los recursos tecnológicos más útiles para presentar el contenido sino más propicios para generar aprendizajes significativos y cambios en la manera de enseñar. Es decir, incluye a la mirada pedagógica didáctica sobre las TIC, sus usos con intenciones y fines exclusivamente educativos. Puesto que la gran mayoría de los programas de software, las aplicaciones digitales, no fueron creados con objetivos educativos, sino con otros fines como comerciales, de entretenimiento, entre otros, es necesario el trabajo del docente sobre los mismos y el conocimiento sobre el contexto en el que se enmarca su propuesta de enseñanza, para promover realmente aprendizajes mejores a sus estudiantes.

TPACK (Saberes tecnológicos y Pedagógicos del Contenido): se refiere a la combinación de los diversos tipos de conocimientos mencionados, que supera ampliamente los rasgos de cada uno en forma particular puesto que, se traduce en modos de enseñar con tecnologías que medien la práctica docente de forma eficiente, en términos de aprendizajes significativos generados en el estudiantado y profesorado. En otras palabras, "la particular amalgama que integra y transforma conocimientos disciplinares, pedagógicos y tecnológicos para su enseñanza" (Flores y Demuth, 2015:143). 
Asimismo, en décadas recientes debido al surgimiento de cambios acelerados en diversas facetas de la sociedad producto de una combinación de factores o causas, económicos, políticos, culturales y tecnológicos el campo educativo fue y es empapado de múltiples teóricas pedagógicas, perspectivas didácticas y epistemológicas.

Un término que en 1980 se permeó en la esfera educativa haciéndose popular fue el de "competencias", a través de la Educación Basada en Competencias (EBC) que promueve un enfoque holístico educativo en el cual el tiempo de aprendizaje es flexible, hay una integración entre experiencias de vida, conocimientos, valores y habilidades que producen determinadas competencias, a su vez, el resultado de aprendizaje es lo fundamental (Everhart, 2014).

El Observatorio de Innovación Educativa (2015) indica que ésta es una integración de conocimientos, actitudes, habilidades y valores que permiten a un sujeto desplegarse en distintos contextos, de manera eficaz y llevar a cabo adecuadamente un rol o actividad. A su vez integra todas las dimensiones del sujeto, como saber, saber hacer, saber ser y estar (Blanco, 2009). Además, no solo se coloca el énfasis en los diseños o programaciones de enseñanza para los estudiantes sino también en la formación docente. Destacamos entonces, en esta línea una reciente reinterpretación del TPACK por parte de Cejas, Navío y Barroso (2016) quienes plantean visualizar el modelo TPACK desde este término. Señalan que éstas "se caracterizan por su carácter teórico-práctico, aplicativo, contextualizado, reconstructivo, combinatorio e interactivo" (Cejas, Navío y Barroso, 2016:109). Dichos autores, plantean superar la articulación de conocimientos que presenta el modelo TPACK original, por el entretejido de competencias docentes desplegadas en la práctica de enseñanza atravesada e integrada curricularmente por las TIC. De esta manera los tres tipos de conocimientos cuyas intersecciones dan lugar a la producción de otros, el conocimiento pedagógico (PK), el conocimiento del contenido (CK) y conocimiento tecnológico (TK), son reinterpretados como competencias pedagógicas (PK), competencias disciplinares (CK), y competencias tecnológicas (TK).

A continuación, se presenta el cuadro que señala estas competencias: 


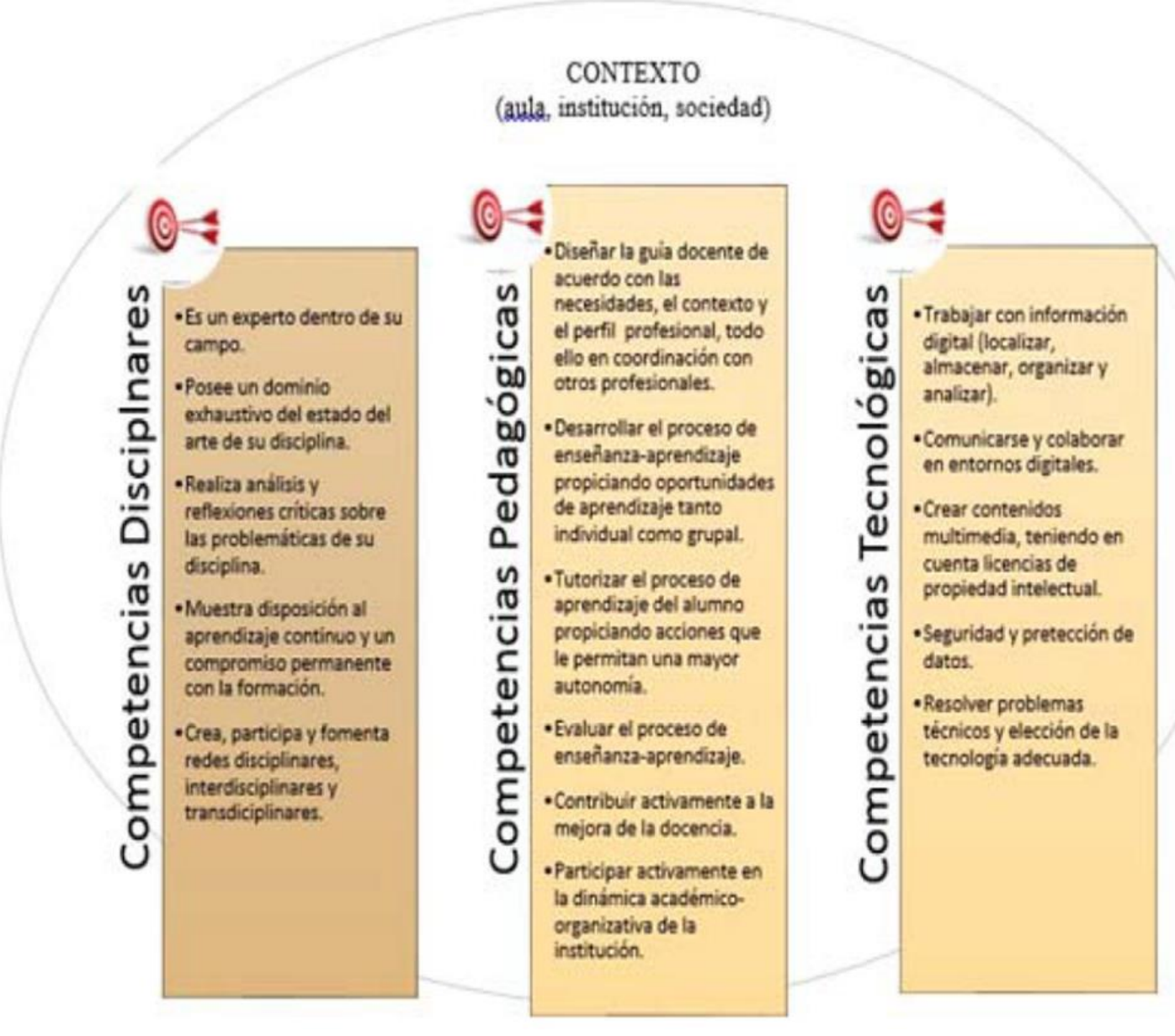

Figura 1.2. Esquema del modelo de competencias del TPACK, organizado a partir de las tres competencias claves y sus características más sobresalientes. Reproducido de "Las competencias del profesorado universitario desde el modelo TPACK (conocimiento tecnológico y pedagógico del contenido)”, p. 110, Cejas L., Navío, A. y Barroso, J. 2016, Pixel-Bit. Revista de Medios y Educación, (49), 105-119.

\section{MÉTODO Y MATERIALES}

Esta investigación se enmarcó en el paradigma interpretativo y enfoque cualitativo. Asimismo, la metodología adoptada es el estudio de casos. Con el propósito de comprender las singularidades de los sujetos y sus experiencias (Stake, 2007), en los procesos de construcción de su conocimiento docente. Este tipo de estudio, según los aportes de Ying (1984) y Martínez Sánchez (2000), coinciden en caracterizarlo como un análisis intensivo, singular, de un tema contextualizado, con una delimitación "natural”, que demanda fuentes de información. Si bien, como sostiene este último, los casos suponen ejemplos concretos no es posible generalizar que las características singulares del contexto universitario de la UNNE y las facultades seleccionadas se reproduzcan en otros sitios estrictamente.

Justamente, "permite el uso de métodos múltiples de recolección para su construcción, los cuales dan lugar a la formulación de interpretaciones de constructores hipotéticamente realistas y a la vez la triangulación de los mismos" (Stake, 2007:99); ésto se relaciona, con las fuentes de 
recolección de información para la metodología de casos, que se utilizó y son estudiadas por Coller (2005): entrevistas (inicial y de profundización), los documentos (planes de estudio, CV, programas de las asignaturas, propuestas de adscripciones) y la observación (de clases orientadas o intervenidas por estudiantes adscriptos).

Para la muestra se trabajó con tres principiantes de las siguientes facultades de la universidad en estudio: Medicina, (carrera de Medicina, asignatura de Bioquímica); Ciencias Exacta, Naturales y Agrimensura (Licenciatura y profesorado en Ciencias Biológicas, asignatura Biología de los Cordados); Humanidades (Licenciatura en Ciencias de la Educación, asignatura Elaboración y Evaluación de Proyectos). Dichos principiantes desempeñaron su rol como adscriptas, entre los periodos lectivos de 2017-2018, se les realizó tres entrevistas en profundidad, se observaron clases impartidas por las mismas y se analizaron documentos curriculares, como sus propuestas de adscripciones y curriculum vitae.

\section{RESULTADOS}

En cuanto a los resultados, teniendo en cuenta los tipos de conocimiento indicados por Mishra y Koheler (2006), párrafos arriba, en los tres casos se reconoció un predominio del CK, las principiantes se focalizaron en el estudio exhaustivo del área disciplinar en el que desarrollaron su adscripción (manejo de principios, conceptos, vinculaciones conceptuales). También se identificó un fuerte anclaje que realizaron en el TK, conocían el manejo y funciones de redes sociales, microscopios, lupas, y aula virtual.

Con respecto al PK también contaban con algunos conocimientos generales sobre la práctica de enseñanza; destacaron el rol docente como fundamental, la disposición de los estudiantes por aprender, los procesos de aprendizajes complejos, las estrategias de enseñanza (conocían la relevancia de la ejemplificación, analogías, uso de anécdotas biográficas, preguntas fácticas y no fácticas, juegos para lograr la interacción con los estudiantes y promover los aprendizajes).

Se reconocieron manifestaciones iniciales que refieren al PCK: el reconocimiento de errores o dificultades frecuentes de los estudiantes que cursaban las asignaturas puesto que, al ser estudiantes que ya aprobaron estas asignaturas señalaban que conocían los errores frecuentes de aprendizaje en el estudio de los temas y los exámenes. Por lo que, avanzaban en presentar otras alternativas en paralelo al desarrollo de los contenidos para que, puedan sortear esos obstáculos o bien atravesarlos, logrando una mayor comprensión y aprendizajes significativos. Ejemplos concretos fueron, el uso de redes sociales utilizadas con el fin de socializar materiales bibliográficos fuera de lo convencional en las asignaturas como videos, tutoriales, artículos científicos, noticias educativas vinculadas a las temáticas tratadas. Asimismo, trabajaron con y desde los ejemplos, analogías, el relato de sus propias experiencias o de pares traducidas en anécdotas biográficas. Intentaron a través de diversas maneras, ideas y recursos disponibles, hacer interesantes los temas tratados en las asignaturas y que los aprendizajes fueran más allá de los contenidos programados en el plan de estudios. 
En cuanto al TCK las principiantes reconocieron en las entrevistas que creían que el uso de redes sociales actuales puede ser potenciales para integrarlas a las propuestas de enseñanza de sus disciplinas porque cuentan con características particulares para mejorar la comunicación con los estudiantes como la inmediatez, simultaneidad, el uso frecuente que estos hacen de ellas, permite el debate, intercambio de materiales o preguntas. A su vez, se vuelven espacios alternativos y en paralelo a las aulas virtuales que según ellas son muy poco frecuentadas por los estudiantes, además de que su acceso es limitado.

Con respecto al TPK éstas inicialmente y de manera paulatina van construyendo la mirada pedagógica didáctica sobre el uso de ciertas tecnologías para integrarlas a sus disciplinas, tomando conciencia poco a poco de que el uso de las tecnologías tiene que fortalecer la comprensión y el aprendizaje profundo y significativo. Se enfatiza que se encuentran en una etapa inicial de construcción el mismo y sobre todo del TPACK que, si bien va desarrollándose paso a paso, todavía no adquiere una forma sólida en las principiantes adscriptas como en el caso de docentes expertos.

\section{DISCUSIÓN}

Este estudio de investigación sostiene que la perspectiva de competencias desde Cejas, Navío y Barroso (2016), es la que emerge en las interpretaciones de los casos, por lo que se toma distancia de la esquematización de conocimientos planteado por Mishra y Koheler (2006). En este sentido, se plantea que, las expresiones iniciales y concretas del CDTC en sus prácticas con integración de TIC (redes sociales, whatapps y Facebook), se manifestó en la interacción y predominio de dos de las CK, como la manifestación de la disposición del aprendizaje continuo que reflejaron en sus prácticas, con un compromiso sostenido sobre la formación y la creación y participación de grupos de estudio; además de PK como promover oportunidades de aprendizaje grupales durante las clases y en horarios extra clases. Asimismo, las TK, al trabajar con información digital, comunicarse, colaborar en entornos virtuales mediante las iniciativas y propuestas de tareas, actividades complementarias a las programadas por las asignaturas.

A partir de lo expuesto, se sostiene que en sus prácticas convergen inicialmente tres componentes de este modelo (TPACK), el entorno virtual (whatsapp, facebook), el conocimiento disciplinar (por medio de videos, papers seleccionados para la comprensión, actividades de aprendizaje) e intervenciones y orientaciones específicas de las principiantes adscriptas para la profundización por parte de los estudiantes de contenidos desarrollados en la presencialidad a través de debates, preguntas críticas y no simplemente fácticas.

Analizar la integración de las TIC en las propuestas de enseñanza y diseñar propósitos didácticos pedagógicos que subyacen a esta articulación, contribuyen a la construcción del conocimiento del estudiante adscripto puesto que, en los tres casos mencionaron distintos recursos utilizados, e integrados a la propuesta curricular, como ser plataforma virtual Moodle, redes sociales como Facebook, WhatsApp, presentaciones de power point, videos, en sus prácticas de adscripción. No solo hay un reconocimiento de que se debe saber el uso operativo, técnico o de funcionamiento de las mismas, sino que se debe descubrir el/ los objetivo/s didáctico/s pedagógico 
/s de su uso y ponerlo en práctica. Es decir, en un primer nivel saber su funcionamiento y en un segundo nivel dar un salto de tipo reflexivo y crítico colocando la impronta de generar o fortalecer los aprendizajes significativos en sí mismo como sujeto y en el otro a partir de su mediación. Ejemplos concretos que ilustran lo planteado anteriormente, es el uso de la red social WhastApp por parte de las principiantes, quienes señalaron que cotidianamente su uso guardaba un fin meramente de entretenimiento y comunicación. Luego, durante su práctica de adscripción y junto a sus demás pares adscriptos decidieron crear grupos con los estudiantes de las clases en esta red social, con un fin educativo. En los mismos compartían videos sobre diversos temas tratados en clase, para generar mayor comprensión de los mismos, revistas electrónicas científicas que contenían artículos contenidos, novedades o noticias actuales sobre los temas de estudio. Suárez Ramos (2017) señala que el uso de TIC en la enseñanza de las ciencias en general, y en particular en las biológicas, hace que los estudiantes se interesen por aprender y conocer más, facilitan la comunicación no presencial aprovechando el tiempo extra-clases.

Además, se señala que estas experiencias ilustran la iniciativa de migración, desde un entorno virtual institucional con acceso limitado para los principiantes, a entornos virtuales más flexibles y de uso cotidiano por las generaciones actuales, que promueven la cercanía con el equipo de asignatura, comunicación al instante, fluida, el acompañamiento y seguimiento tutorial.

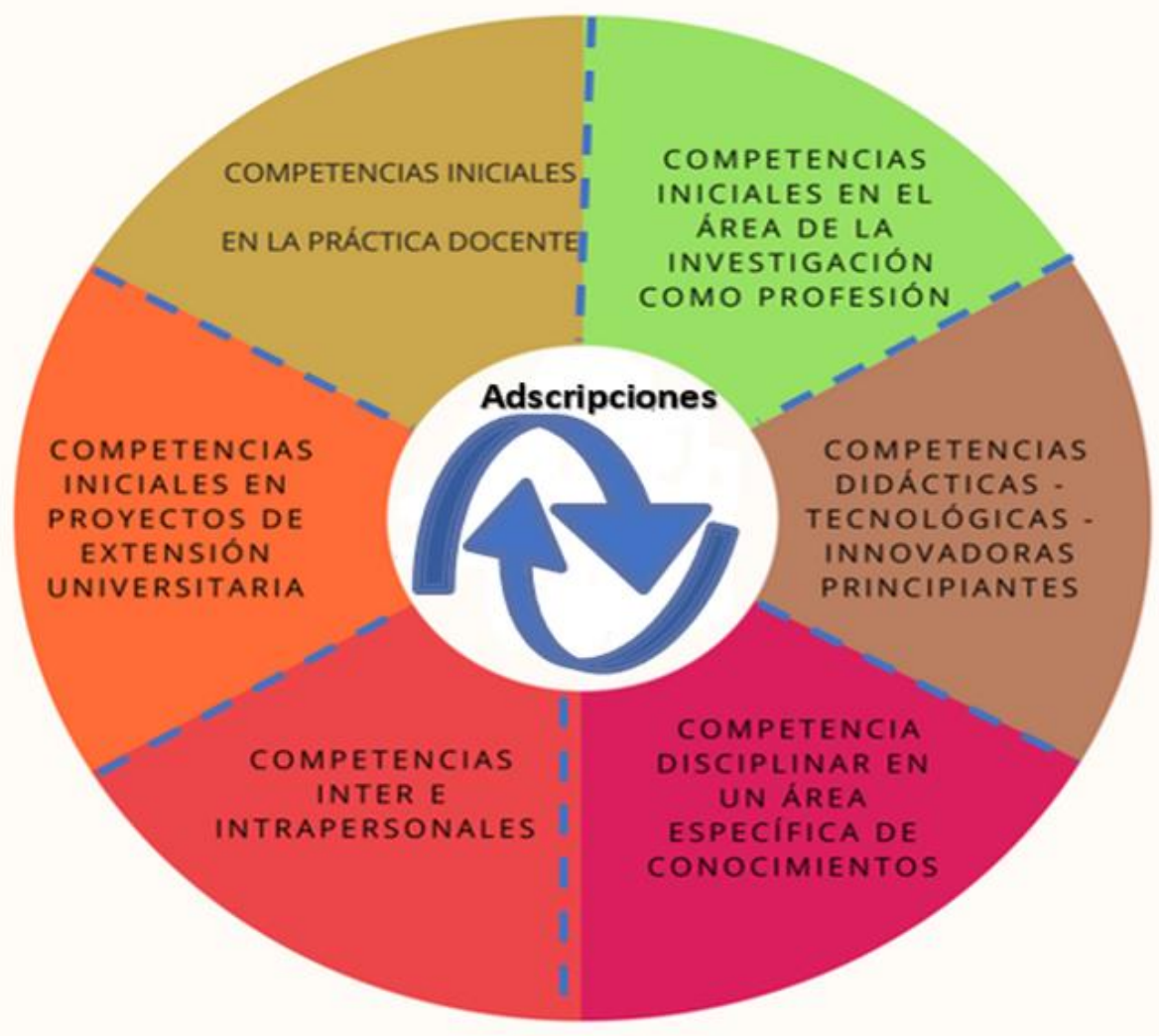

Figura 1.3. Esquema de integración sobre las seis competencias iniciales del estudiante adscripto. 


\section{CONCLUSIONES}

Al indagar qué sucede con el conocimiento docente en los inicios de la formación en un principiante que atraviesa por el espacio de una adscripción académica, se plantea que al ser éste tan diverso en cuanto a experiencias que pueden desplegarse en su interior (experiencias de prácticas docentes, tutorías, de investigación, extensión universitaria, proyectos) hace que, en la construcción de su conocimiento, converjan distintos saberes, que permiten desplegar una serie de competencias en los principiantes. Asimismo, estas competencias se vinculan con el CDTC puesto que, depende cuál de ellas se desarrolle más exhaustivamente, éste se va perfilando y delineando inicial y paulatinamente nutriéndose de éstas. Dichas competencias se interrelacionan, retroalimentan y fusionan entre sí. Estas se agrupan en convencionales, aquellas que son propias del rol porque se basan en los reglamentos institucionales que regulan las funciones de los adscriptos y las emergentes que nacen a partir de la práctica de enseñanza cotidiana y en contacto diario con los grupos de estudiantes que se encuentran en un contexto atravesado por las TIC.

Competencias convencionales:

a.- Competencia disciplinar en un área específica de conocimientos: el principiante adscripto con el propósito de lograr claridad y solvencia en las explicaciones que brinda a los estudiantes de un curso, ante dudas, preguntas o dificultades que estos presentan, profundiza la lectura y estudio de los marcos teóricos dados y logra una mayor comprensión disciplinar.

b-. Competencias iniciales en la práctica docente: el principiante adscripto se ocupa de la detección y reconocimiento de errores frecuentes en los procesos de aprendizajes al estudiar un tema, o preparase para exámenes parciales o finales y los tiene en cuenta cuando realiza orientaciones sobre técnicas de estudio, clases extras de tutoría en las cuales realiza un seguimiento y acompañamiento, el desarrollo de micro-clases, en las cuales diseña una propuesta de enseñanza sobre un contenido disciplinar, trabaja con estrategias de enseñanza e implementa el uso de recursos tecnológicos.

c-. Competencias iniciales en el área de la investigación como profesión: en algunos casos, el adscripto incursiona en el espacio de la adscripción para vincularse con el área de la investigación y poder comprender este campo de conocimientos, indagando, explorando y practicando técnicas, estrategias, metodologías en relación con el área disciplinar en el que se encuentra. Pero no solo para comprender, conocer y adquirir competencias y experiencias, sino que, lo visualiza como un posible itinerario profesional a futuro.

d-. Competencias en desarrollar actividades de extensión universitaria: en casos particulares, el adscripto se vincula estrechamente con la misión social de la universidad desplegando y aprendiendo una serie de competencias vinculadas a la solidaridad, generosidad, compromiso y responsabilidad de trabajar en conjunto con los diversos actores sociales del medio, para mejorar la calidad de vida. Competencias sociales que fortalecen las relaciones intra e interpersonales, como la detección de falencias, problemas crisis en las instituciones con las cuales deben trabajar, la gestión, toma de decisión y colaboración en los grupos de trabajos.

Competencias emergentes:

e-. Competencias didácticas -tecnológicas -innovadoras: Si bien es cierto que los jóvenes actuales, por el contexto sociocultural y tecnológico mundial, poseen características, conocimientos y manejo permanente de recursos tecnológicos muy distintos a generaciones pasadas, necesitan guía 
y orientación didáctica sobre el acceso y los usos de dichos recursos, para ir trabajando su formación académica, profesional, personal y ética. En este sentido, se destaca por parte del principiante adscripto, la implementación y desarrollo de estrategias de enseñanza en entornos virtuales como redes sociales (Facebook, WhatsApp), permitiéndole realizar un acompañamiento más estrecho a los estudiantes. Éste, como operador de las nuevas tecnologías en la asignatura logra mejorar la comunicación con los estudiantes y el equipo de asignatura ya que acorta las distancias jerárquicas entre ambos. Si bien hacen uso de recursos tecnológicos cotidianos para los estudiantes, trabaja con un fin didáctico sobre ellos, a partir de los conocimientos que cuentan y trata de intégralos con el conocimiento disciplinar que enseñan en las asignaturas, para generar aprendizajes de largo alcance.

f-. Competencias inter e intrapersonales: Los vínculos estrechos entre pares que se generan por diversas situaciones como: compartir el mismo espacio de formación, el acompañamiento constante de los adscriptos al grupo clase, a través de tutorías, redes sociales, micro-clases, orientaciones sobre las evaluaciones, permite el reconocimiento de valores como el respeto, tolerancia, paciencia y e influencia en la gestación de competencias interpersonales como la empatía, el trabajo en equipo, fluidez en la oratoria y enseñanza. permite el incentivo de competencias intrapersonales como la estabilidad emocional, el espíritu de servicio y ayuda al otro, la creatividad, seguridad.

Se resalta que, en tres perfiles profesionales diversos se identificó a la adscripción como un espacio multidimensional en cuanto a experiencias que pueden desplegarse en su interior como las prácticas docentes, de investigación y extensión universitaria. Esto permite que en la construcción del conocimiento del neófito converjan distintos saberes y una serie de competencias que se interrelacionan, retroalimentan y fusionan entre sí, permitiendo el desarrollo de competencias convencionales y el surgimiento de otras, las emergentes.

Finalmente, se señala que las experiencias de adscripciones al ser tan particulares y personales permiten enriquecer la formación del principiante universitario en algún área específica, ya sea en el área de la docencia, de la investigación, o extensión universitaria, dependiendo de los intereses e intenciones individuales que se posea. Además, posibilita el despliegue de ese arsenal de competencias iniciales en la formación de pregrado, que diferencia la práctica profesional de un estudiante que decidió transitar esta experiencia de aquel que nunca lo hizo. También, da lugar a conocer y conocerse, acerca de gustos, preferencias y pasiones por áreas disciplinares teniendo en cuenta que algunas carreras son tan diversas en cuanto a los contenidos disciplinares que trata, como son los casos de medicina, Biología o Ciencias de la Educación; aporta entonces, claridad al respecto y focalización sobre el futuro desempeño profesional que se descubre y desea.

Este espacio contribuye a la construcción de sus conocimientos puesto que, propicias experiencias que permiten emerger competencias y habilidades particulares en el principiante.

La adscripción también se convierte en un espacio que contiene y acompaña al principiante durante sus primeras experiencias en la práctica docente, ya que, los primeros años de éste es considerado caótico, conflictivo emocionalmente, por las cargas y presiones que implica insertarse en un departamento o equipo docente que se mueve en una cultura institucional particular, en el cual los códigos, relaciones, tratos, formas de ser y hacer son naturalizadas por aquellos docentes con mayor experiencia que se encuentran en el ámbito. 
Por último, se presenta un esquema (ver Figura 1.3) de las competencias mencionadas, como producto del estudio de investigación realizado, en el cual es posible visualizar la integración entre ellas durante una instancia de formación como la adscripción universitaria.

\section{REFERENCIAS BIBLIOGRÁFICAS}

Blanco, A. (2009). Desarrollo y Evaluación de Competencias en Educación Superior. España: Narcea.

Briones, G. (2001). Tendencias recientes de la investigación en pedagogía. Áreas, problemas y formas de relación. Revista la sociología en sus escenarios, 5(4), 73-88.

Cabero, A., J., y Marín, D. (2017). La educación formal de los formadores de la era digital - los educadores del siglo XXI. IDUS depósito de Investigación Universidad de Sevilla, 44, 29-42. Recuperado de http://hdl.handle.net/11441/59053.

Cabero A., J., y Marín D., y Castaño G. (2015). Validación de la aplicación del modelo TPACK para la formación del profesorado en TIC. @ tic. revista d'innovació educativa, (14), 13 22.

Cano, E. (2005). Cómo mejorar las competencias de los docentes. Guía para la autoevaluación y el desarrollo de las competencias del profesorado. Profesorado: Revista de curriculum y formación del profesorado 8(2), 1-12.

Cejas, R., Navío A., y Barroso, J. (2016). Las competencias del profesorado universitario desde el modelo tpack (conocimiento tecnológico y pedagógico del contenido). Pixel-Bit. Revista de Medios y Educación, 49, 105-119.

Coller, X. (2005). Estudio de casos. Madrid: Centro de investigaciones sociológicas.

Cukierman U., y Virgili, J. (2013). La educación en el siglo XXI: Los desafíos de la Era Digital.III Jornada de Enseñanza en la Ingeniería. Rectorado Universidad Tecnológica Nacional.

Everhart, D. (2014). Advancing Competency Based Education with the American Council on Education. Blackboard Blog. 5, 6-37.

Ferreiro, J., y Lynch, I. (2015). El valor formativo de la experiencia de la adscripción a la docencia en los profesorados universitarios: relatos de una experiencia de aproximación a las prácticas profesionales docente en la cátedra política, organización y gestión educativa del Departamento de Educación Cientifica FCEYN UNMDP. Recuperado de Actas de Congreso de VIII Jornadas Nacionales y 1er Congreso Internacional sobre la Formación del Profesorado. Mar del Plata.

Flores, F., y Demuth, P. (2015). Construcción del Conocimiento Didáctico-Tecnológico del Contenido en equipos de asignaturas universitarias. Revisión de la literatura. X Congreso sobre Tecnología en Educación \& Educación en Tecnología. Red de Universidades con Carreras en Informática (RedUNCI). (TE\&ET). ISBN: 978-950-656-154-3.

Koehler, M., \& Mishra, P. (2008). Introducing TPACK. In AACTE Committee on Innovation \& Technology (Eds.), Handbook of technological pedagogical content knowledge for educators. 3-29. New York: Routledge.

Koehler, M., Mishra, P., \& Yahya, K. (2007). Tracing the development of teacher knowledge in a design seminar: Integrating content, pedagogy and technology. Computers \& Education, $49,740-762$. 
Koehler, M. \& Misrha, P. (2006). Technological pedagogical content knowledge: Aframework for integrating technology in teacher knowledge. Teachers College Record, 118(6), 10171054.

Koehler, M. \& Mishra, P. (2005). Teachers Learning Technology by Design. Journal of Computing in Teacher Education, 21(3), 94-102. Retrieved October 16, 2019 from https://www.learntechlib.org/p/55262/.

Martínez, A. (2000). El estudio de casos como estrategia didáctica para la formación de profesionales de la acción social. El estudio de casos: para profesionales de la acción social. Madrid: Narcea.

Medina M., y Jarauta-Borrasca, B. (2013). Análisis del conocimiento didáctico del contenido de tres profesores universitarios. Revista de educación, 360, 600-623.

Montero. L. (2001). La construcción del conocimiento profesional docente. Rosario: Homo Sapiens.

Observatorio de Innovación educativa del Tecnológico de Monterrey (2015). Reporte Edu Trends. Educación Basada en Competencias. Recuperado en: http://www.redage.org/sites/default/files/adjuntos/edu_trends_ebc.pdf

Ronderos, P y Valderrama, A. (2003). El Futuro de la Tecnología: una aproximación desde la historiografía. Revista Iberoamericana de Ciencia, Tecnología, Sociedad e Innovación . 5.

Schmidt, D., Baran, E., Thompson, A., Mishra, P., Koehler, M., \& Shin, T. (2009). Technological pedagogical content knowledge (TPACK): The development and validation of an assessment instrument for preservice teachers. Journal of Research on Technology in Education, 42(2), 123-149.

Shulman, L. (1986). Paradigms and research programs in the study ofteaching: a contemporary perspective. In: WITTROCK, M. (Ed.) Handbook ofResearch on Teaching. 3 (ed) 3-36 New York: Macmillam. Publishing Company.

Shulman, L. (1987). Knowledge and Teaching: Foundations of the New Reform. Harvard Educational Review 57(1), 1-22.

Solberg, V., Rodríguez, G., Ulloa, G. y Viñas, W. (2007). El adscripto... ¿quién? Conceptualizaciones sobre un rol poco abordado. En V Encuentro Nacional y II Latinoamericano La Universidad como objeto de investigación. Tandil. Argentina.

Stake, R. (2007). Investigación con estudios de caso. Madrid, Morata.

Suárez, R. (2017). Importancia del uso de recursos didácticos en el proceso de enseñanza y aprendizaje de las ciencias biológicas para la estimulación visual del estudiantado. Revista Electrónica Educare (Educare Electronic Journal), 21(2),1-18.

Torres-Gastelú, C., y Arras V. (2011). Percepción de estudiantes de educación superior sobre competencias en las TIC en las universidades Autónoma de Chihuahua y Veracruzana. Apertura, 3(2), 1-19.

Ying, R. (1984). Case Study Research: Design and Methods, Applied social research Methods Series, Newbury Park CA, Sage. 